UOSTP 5101

OU-HET 544

hep-th/0511051

\title{
M-theory Supertubes with Three and Four Charges
}

\author{
Dongsu Bak, ${ }^{a}$ Kyungyu Kim ${ }^{b}$ and Nobuyoshi Ohta ${ }^{c}$ \\ a Physics Department, University of Seoul, Seoul 130-743, Korea \\ ${ }^{b}$ School of Physics, Seoul National University, Seoul 151-747, Korea \\ ${ }^{c}$ Department of Physics, Osaka University, Toyonaka, Osaka 560-0043, Japan \\ (dsbak@mach.uos.ac.kr, ohta@phys.sci.osaka-u.ac.jp)
}

\begin{abstract}
Using the covariant M5-brane action, we construct configurations corresponding to supertubes with three and four charges. We derive the BPS equations and study the full structure of the solutions. In particular, we find new solutions involving arbitrariness in field strengths.
\end{abstract}




\section{Introduction}

The counting of microstates associated with the black holes in superstring theories is a subject of current interest. The configurations are called BPS solutions which preserve part of supersymmetry, and this is the key property in the above counting of microstates. Among others, the BPS configurations called supertubes [1]-[29] has a dual realization in the brane worldvolume as well as supergravity. The entropy obtained for the latter is beautifully explained in the microstate counting in the former realization. This is especially successful in the supertubes with 2 charges, which is realized as a round D2-brane configuration of tubular shape involving D0 and F1 on the worldvolume. (For other related discussions, see Refs. [30]-[34].)

There are also more interesting solutions corresponding to supertubes with 3 and 4 charges, which have been less understood [25]-[28]. The configurations may have various forms of realization involving three branes in addition to certain numbers of dipole charges, which may be related by duality transformations. So it is convenient to consider the configurations in the context of M-theory. In this paper, we construct these solutions in the M-theory using the covariant action of M5-branes [35] and study their properties. To discuss this class of solutions, we have to study the BPS equations whose solutions are the objects of our interest.

The configurations we consider for 3-charge case can be schematically written as

\begin{tabular}{|l|llllllll|}
\hline M2 & 1 & 2 & & & & & \\
M2 & & & 3 & 4 & & & \\
M2 & & & & & 5 & 6 & \\
m5 & 1 & 2 & 3 & 4 & & & $\theta$ \\
m5 & 1 & 2 & & & 5 & 6 & $\theta$ \\
m5 & & & 3 & 4 & 5 & 6 & $\theta$ \\
\hline
\end{tabular}

where M2 stands for M2-branes lying in (12), (34) and (56) spatial directions within the "round" M5-branes in $(1234 \theta),(1256 \theta),(3456 \theta)$ directions where 1-6 refer to the directions in the tangent space. Later we shall find that the shape of $m 5$ can be much more general than the above. The circular direction of the M5-branes is parametrized by the angle $\theta$ in the (789 4 ) directions of the target space ( $\downarrow$ stands for 10). The total charges of these M5-branes are zero and for this reason they are denoted as m5. The M2-branes can be understood as induced by the background fields on the worldvolume of m5-branes.

If we put one of the M2-brane charges to zero, say the third one, we could forget about the second and third m5-branes, and get M-theory realization of supertubes with 2 charges (see for 
instance [10]).

Using the covariant action for M5-branes, we make a detailed analysis of 3-charge configurations (1) and briefly discuss the extension to 4-charge case. In section 2, we derive the BPS equations from the kappa symmetry in the M5-brane action [35]. In section 3, we work out the solutions explicitly. Specifically we consider the case in which the worldvolume of $\mathrm{m} 5$ is $T^{4}$ which is embedded inside the target space of $T^{6}$. This configuration is also discussed in Ref. [26], but our construction is more explicit and contains new solutions involving three arbitrary functions of $\theta$. In section 4, we derive the conserved charges of the system and discuss the bound on the curve imposed by these charges. This should be useful for the counting of the microstates for fixed conserved charges [20]. In section 5, we consider the embedding of m5 and M2 system into a Calabi-Yau (CY) 3-fold instead of $T^{6}$, and repeat the similar analysis of the BPS solutions. One of the resulting BPS equations is a nonlinear instanton equation [39] whose quadratic fluctuations for the degeneracy counting and understanding the related fluctuations is briefly touched upon. In section 6 , we briefly discuss the generalization to the case of four M2-charges in the tangent space of $T^{8}$ or CY 4-fold. Section 7 is devoted to conclusion.

\section{BPS equations}

We aim to construct the configuration (1) with the covariant action of M5-brane proposed in Ref. [35], and use the same notation. In particular, we should note that the flat metric is chosen as $\eta_{a b}=\operatorname{diag}(+1,-1,-1, \ldots)$.

The worldvolume of $\mathrm{m} 5$ is parametrized by $\left(t, \sigma_{i}, \theta\right),(i=1,2,3,4)$, its embedding into the flat target space is given by $\left(T, Y^{a}\left(\sigma_{i}\right)\right),(a=1, \ldots, 6)$, and the m5-brane curve in the $(7,8,9, \sqsubset)$ space is denoted by $X^{m}(\theta),(m=7,8,9, \natural)$. We fix the gauge $t=T$. The induced vielbein on the worldvolume

$$
e_{i}^{a}=\frac{\partial Y^{a}}{\partial \sigma^{i}}
$$

relates the gamma matrices as

$$
\gamma_{i}=e_{i}^{a} \Gamma_{a}=\partial_{i} Y^{a} \Gamma_{a}, \quad \gamma_{0}=\Gamma_{0}, \quad \gamma_{5}=X^{m \prime} \Gamma_{m},
$$

and the induced metric

$$
g_{00}=1, \quad g_{i j}=\partial_{i} Y^{a} \partial_{j} Y_{a}, \quad g_{55}=X^{m \prime} X_{m}^{\prime},
$$

where 5 denotes $\theta$, the 5 -th worldvolume coordinate, and the prime is its derivative. 
Let us now find out the BPS equations. They should be derived from the kappa symmetry [35], which gives

$$
(\Gamma+1) \epsilon=0
$$

where

$$
\begin{aligned}
& \Gamma=\frac{\sqrt{-g}}{\sqrt{-\operatorname{det}(g+i H)}} {\left[\frac{\gamma^{(6)}}{\sqrt{-g}}+\frac{i}{2 \sqrt{-(\partial a)^{2}}} H^{m n} \gamma_{m n} \gamma_{p} \partial^{p} a\right.} \\
&\left.+\frac{\sqrt{-g}}{8(\partial a)^{2}} \partial^{m_{1}} a \epsilon_{m_{1} \ldots m_{6}} H^{m_{2} m_{3}} H^{m_{4} m_{5}} \gamma^{m_{6}} \gamma_{p} \partial^{p} a\right]
\end{aligned}
$$

where $\epsilon^{012345}=-\epsilon_{012345}=1$, and $g$ is the determinant of the five-dimensional induced metric.

We expect the solution is given by the projection

$$
\epsilon=P \epsilon_{0} ; \quad P=P_{1} P_{2} P_{3}
$$

where $P_{1}=\frac{1+i \Gamma_{012}}{2}, P_{2}=\frac{1+i \Gamma_{034}}{2}, P_{3}=\frac{1+i \Gamma_{056}}{2}$ are the projectors. We choose our gauge for $a$ as

$$
a=t
$$

so that $(\partial a)^{2}=1$. Our task is now to examine what conditions on the fields we get from (5).

The first term in (6) involves

$$
\gamma^{(6)}=\gamma_{0} \ldots \gamma_{5}=\Gamma_{0} \gamma_{5} \Gamma_{a b c d} f^{a b c d}
$$

where we have defined

$$
f^{a b c d}=\frac{1}{4 !} \epsilon^{i j k l} e_{i}^{a} e_{j}^{b} e_{k}^{c} e_{l}^{d}
$$

The term (9) produces two different kinds of terms:

$$
f^{a b c d} \Gamma_{a b c d} P=-\frac{1}{2} f^{a b c d} \epsilon_{a b c d e f} \epsilon^{e f} P-3 \epsilon^{a b}\left(f^{a b c e} \epsilon^{e d}-f^{a b d e} \epsilon^{e c}\right) \Gamma_{c d} P,
$$

depending on whether $(a, b, c, d)$ belong to two sets out of (12),(34) and (56), or $c$ and $d$ belong to different sets other than $(a, b)$. Here we have used the projection condition $(7)$ and defined $\epsilon^{a b}$ as $6 \times 6$ matrix:

$$
(\epsilon)^{a b} \equiv\left(\begin{array}{ccc}
I & 0 & 0 \\
0 & I & 0 \\
0 & 0 & I
\end{array}\right) ; I \equiv\left(\begin{array}{cc}
0 & 1 \\
-1 & 0
\end{array}\right)
$$


The second term in (6) is decomposed as

$$
\frac{1}{2}\left[2 f^{(05)} \gamma_{0} \gamma_{5}+2 f^{(0) a} \gamma_{0} \Gamma_{a}+2 f^{(5) a} \gamma_{5} \Gamma_{a}+f^{a b} \Gamma_{a b}\right] \gamma_{0},
$$

where we have defined

$$
f^{a b}=H^{i j} e_{i}^{a} e_{j}^{b}, \quad f^{(0) a}=H^{0 i} e_{i}^{a}, \quad f^{(5) a}=H^{5 i} e_{i}^{a}, \quad f^{(05)}=H^{05}
$$

The first three terms in eq. (13) are rewritten as

$$
-\gamma_{5} f^{(05)}-\Gamma_{a} f^{(0) a}+\Gamma_{0} \gamma_{5} \Gamma_{a} f^{(5) a},
$$

and the last term yields

$$
-\frac{i}{2} \epsilon_{a b} f^{a b} P+\frac{1}{4} \Gamma_{0} \Gamma_{a b}\left(f^{a c} \epsilon^{c b}-f^{b c} \epsilon^{c a}\right) P .
$$

Finally we come to the third term in (6). We find that they give

$$
\begin{aligned}
& -\frac{\sqrt{-g}}{8}\left[\epsilon_{i j k l} H^{i j} H^{k l} g^{55} \gamma_{5}+4 \epsilon_{i j k l} H^{j k} H^{l 5} \gamma^{i}\right] \Gamma^{0} \\
= & -\frac{\sqrt{-g}}{8}\left[\left|g^{55}\right| \epsilon_{i j k l} H^{i j} H^{k l} \Gamma_{0} \gamma_{5}+4 \epsilon_{i j k l} H^{j k} H^{l 5} g^{i m} e_{m}^{a} \Gamma_{a} \Gamma_{0}\right] \\
= & -\frac{\sqrt{-g}}{8}\left[\left|g^{55}\right| \epsilon_{i j k l} H^{i j} H^{k l} \Gamma_{0} \gamma_{5}-i 4 \epsilon_{i j k l} H^{j k} H^{l 5} g^{i m} e_{m}^{a} \Gamma_{b} \epsilon_{a b}\right] .
\end{aligned}
$$

We have the $\gamma_{5}$ term only in the first term in (15) to obtain

$$
f^{(05)}=0
$$

and $\Gamma_{0} \gamma_{5}$ terms only from the first term in (11) and (17), so

$$
\frac{1}{2} \epsilon_{a b c d e f} f^{a b c d} \epsilon^{e f}+\frac{|g|}{8}\left|g^{55}\right| \epsilon_{i j k l} H^{i j} H^{k l}=0 .
$$

The second term in (11) is the only $\gamma_{5} \Gamma_{c d}$ term, so we must have

$$
\epsilon^{a b}\left(f^{a b c e} \epsilon^{e d}-f^{a b d e} \epsilon^{e c}\right)=0 .
$$

The second term in (15) and the last term in (17) involving $\Gamma_{a}$ give

$$
f^{(0) a}-\frac{i}{2} \sqrt{-g} \epsilon_{i j k l} H^{j k} H^{l 5} g^{i m} e_{m}^{b} \epsilon_{b}^{a}=0 .
$$

The third term in (15) gives

$$
f^{(5) a}=0,
$$


and so $H^{l 5}=0$. Combined with (21), this gives

$$
f^{(0) a}=0 .
$$

The last term in (16) gives

$$
f^{a c} \epsilon^{c b}-f^{b c} \epsilon^{c a}=0
$$

Finally the first term in (16) should cancel 1:

$$
-\frac{i}{2} \epsilon_{a b} f^{a b}+\frac{\sqrt{-\operatorname{det}(g+i H)}}{\sqrt{-g}}=0 .
$$

We have thus derived all the BPS equations resulting from the kappa symmetry. They are eqs. $(18)-(25)$. We are now going to find solutions to these equations.

\section{BPS solutions}

To solve our BPS equations, we first make further gauge choice

$$
\left(Y^{1}, Y^{2}, Y^{3}, Y^{4}\right)=\left(\sigma^{1}, \sigma^{2}, \sigma^{3}, \sigma^{4}\right) .
$$

Let us first examine (20). Defining the complex coordinates

$$
Z=Y^{5}+i Y^{6} ; \quad w_{1}=\sigma^{1}+i \sigma^{2}, \quad w_{2}=\sigma^{3}+i \sigma^{4},
$$

and writing explicitly the indices, we find

$$
f^{1,2,3+i 4,5+i 6}=f^{3,4,1+i 2,5+i 6}=f^{5,6,1+i 2,3+i 4}=0 .
$$

Using the definition (10), we find that (28) is equivalent to

$$
\partial_{\bar{w}_{1}} Z=\partial_{\bar{w}_{2}} Z=0 \text {. }
$$

(The last equality in (28) follows from these relations.)

Next (24) can be rewritten as $H^{w_{1} w_{2}}=H^{w_{1} j} \partial_{j} Z=H^{w_{2} j} \partial_{j} Z=0$. The latter two conditions follow from $(29)$ and $H^{w_{1} w_{2}}=0$, so the first condition

$$
H^{w_{1} w_{2}}=0
$$

is the only relevant relation. We note that this can be also written as

$$
H^{13}=H^{24}, \quad H^{14}=H^{32} .
$$

On the other hand, (18), (22) and (23) mean that

$$
H^{50}=H^{5 i}=H^{0 i}=0 .
$$




\subsection{General analysis of BPS equations}

To examine the remaining conditions (19) and (25), we need the induced metric (4):

$$
g_{i j}=-\delta_{i j}-\frac{1}{2}\left(\partial_{i} Z \partial_{j} \bar{Z}+\partial_{j} Z \partial_{i} \bar{Z}\right)
$$

With the help of (29), one may show that $g_{12}=0, g_{34}=0$ and

$$
g_{11}=g_{22}, \quad g_{33}=g_{44}, \quad g_{13}=g_{24}, \quad g_{14}=-g_{23} .
$$

Also by a direct computation, it is straightforward to show that

$$
g_{(4)}=\operatorname{det} g_{i j}=\left(g_{11} g_{33}-g_{13}^{2}-g_{23}^{2}\right)^{2}=(1+\nabla Z \cdot \nabla \bar{Z} / 2)^{2} .
$$

Then (19) can be cast into

$$
\frac{1}{8} \sqrt{g_{(4)}} \epsilon_{i j k l} H^{i j} H^{k l}=-1
$$

One also has

$$
-\operatorname{det}(g+i H)=\left|g_{55}\right| g_{(4)}\left[1-\frac{1}{2} H_{i j} H^{i j}+\frac{1}{8^{2}}\left(\sqrt{g_{(4)}} \epsilon_{i j k l} H_{i j} H_{k l}\right)^{2}\right],
$$

which may be rearranged to

$$
-\frac{\operatorname{det}(g+i H)}{|g|}=\left(1+\frac{\sqrt{g_{(4)}}}{8} \epsilon_{i j k l} H^{i j} H^{k l}\right)^{2}-\left(\frac{1}{2} H_{i j} H^{i j}+\frac{\sqrt{g_{(4)}}}{4} \epsilon_{i j k l} H^{i j} H^{k l}\right) .
$$

Using the properties of $g_{i j}$, the term in the second parenthesis may be written as

$$
\begin{aligned}
& \left\{H^{12} g_{11}+H^{34} g_{33}-\left(H^{13}+H^{24}\right) g_{14}+\left(H^{14}+H^{32}\right) g_{13}\right\}^{2} \\
& +\left\{\left(H^{13}+H^{42}\right)^{2}+\left(H^{14}+H^{23}\right)^{2}\right\}\left(g_{11} g_{33}-g_{13}^{2}-g_{14}^{2}\right)
\end{aligned}
$$

whose second term vanishes due to (31). Since the first term in (38) is zero due to (36), the BPS equation (25) reduces to

$$
\frac{\sqrt{-\operatorname{det}(g+i H)}}{\sqrt{-g}}=-i\left(H^{12} g_{11}+H^{34} g_{33}-2 H^{13} g_{14}+2 H^{14} g_{13}\right)=\frac{i}{2} \epsilon^{a b} f^{a b} .
$$

Using (29) and (33), it is easy to check that this is automatically satisfied.

To summarize, our BPS equations are eqs. (29), (31), (32) and (36) which can be written as

$$
\left(1+\frac{|\nabla Z|^{2}}{2}\right)\left(H^{12} H^{34}-\left(H^{13}\right)^{2}-\left(H^{23}\right)^{2}\right)=-1 .
$$


There is still an equation one has to satisfy [36, 37]:

$$
H_{m n p} \partial^{p} a=V_{m n}
$$

where $V_{m n}$ is defined by

$$
V_{m n}=-2 \frac{\sqrt{-(\partial a)^{2}}}{\sqrt{-g}} \frac{\partial \sqrt{-(g+i H)}}{\partial H^{m n}}
$$

Also $H^{m n}$ is defined by

$$
H^{m n}=\frac{1}{3 ! \sqrt{-g} \sqrt{-(\partial a)^{2}}} \epsilon^{m n l p q r} H_{p q r} \partial_{l} a
$$

from which the equation, $H^{i 0}=H^{50}=0$, follows with the gauge choice $a=t$.

In the present case, one has

$$
H^{i j}=-\frac{i}{2 \sqrt{-g}} \epsilon^{i j k l} H_{k l 5},
$$

and $V^{i j}$ is given by

$$
V_{i j}=\frac{i \sqrt{-g}}{\sqrt{-(g+i H)}}\left(H_{i j}+\frac{1}{2} \sqrt{g_{(4)}} \epsilon_{i j k l} H^{k l}\right) .
$$

Using (40), one finds

$$
F_{i j} \equiv H_{i j 0}=\frac{\sqrt{g_{(4)}}\left(h_{i j}+\frac{1}{2} \sqrt{g_{(4)}} \epsilon_{i j k l} h^{k l}\right)}{\left(g_{11} h_{34}+g_{33} h_{12}+2 g_{14} h_{13}+2 g_{13} h_{41}\right)},
$$

where we have defined

$$
h_{i j}=H_{i j 5} / \sqrt{\left|g_{55}\right|} .
$$

From this and (31), one may show that

$$
\begin{aligned}
& F_{12}=g_{11}, \quad F_{34}=g_{33}, \\
& F_{13}=F_{24}=-g_{14}, \quad F_{14}=F_{32}=g_{13} .
\end{aligned}
$$

This may be succinctly written as

$$
F_{i j}=\frac{1}{2}\left(\epsilon_{i k} g_{k j}-\epsilon_{j k} g_{k i}\right),
$$

which is proportional to the Kähler two form. Introducing the Kähler form $k=g_{p \bar{q}} d w^{p} \wedge d \bar{w}^{\bar{q}}$, we get $F=F_{p \bar{q}} d w^{p} \wedge d \bar{w}^{\bar{q}}=i k$ where $p, q, r=1,2$ and $\bar{p}, \bar{q}, \bar{r}=1,2$ denoting respectively 
the holomorphic and antiholomorphic indices of the m5 worldvolume. The Bianchi identity is satisfied since the Kähler form is closed, i.e. $d k=0$.

In addition, using (31), one may show that

$$
h_{13}=h_{24}, \quad h_{14}=h_{32} \text {. }
$$

Finally, one may consider more general ansatz where $Y^{a}$ 's are also dependent on $\theta$, i.e. $Y^{a}\left(\sigma_{i}, \theta\right)$ and $X^{m}(\theta)$. The induced metric in the 5 space including the $\theta$ direction may be written as

$$
d s_{(5)}^{2}=g_{i j} d \sigma^{i} d \sigma^{j}+2 g_{i 5} d \sigma^{i} d \theta+g_{55} d \theta^{2}
$$

where

$$
g_{i j}=\partial_{i} Y^{a} \partial_{j} Y_{a}, \quad g_{i 5}=\partial_{i} Y^{a} Y_{a}^{\prime}, \quad g_{55}=X^{m \prime} X_{m}^{\prime}+Y^{a \prime} Y_{a}^{\prime}
$$

The similar analysis of BPS equations goes through in this case too. With the gauge choice of (26), $Z$ should be holomorphic function of $w^{p}$ and $H^{w^{1} w^{2}}=0$ with $H^{0 i}=H^{05}=H^{i 5}=0$. Considering the three form, $H_{i j k}=0$ follows from the relation $H^{i 5}=0$. We introduce a two form in the four space by $\tilde{h}_{i j}=H_{i j 5}$. Then $d \tilde{h}=0$ due to the Bianchi identity of the three form field.

Eq. (42) implies $F_{i j 0} d \sigma^{i} \wedge d \sigma^{j}=i g_{p \bar{q}} d w^{p} \wedge d \bar{w}^{\bar{q}}, F_{p 50}=i g_{p 5}$ and $F_{\bar{p} 50}=-i g_{\bar{p} 5}$. With these expressions, the Bianchi identity for the three form field can be checked. The final BPS equation is given by

$$
\frac{1}{8} \epsilon^{i j k l} \tilde{h}_{i j} \tilde{h}_{k l}=G_{55} \sqrt{g_{(4)}},
$$

where $G_{55}=\left|g_{55}-g^{i j} g_{i 5} g_{j 5}\right|$. Though interesting, we will not analyze this generalization further in this note.

\section{$3.2 \quad$ A simple case}

Let us pause to discuss the simple case of $Z=0$. We have $g_{i j}=-\delta_{i j}$. From the BPS equations, there are following set of simple solutions. By eq. (51), we set

$$
h_{13}=h_{24}=a(\theta), \quad h_{14}=h_{32}=b(\theta), \quad h_{12}=c(\theta),
$$

where $a, b$ and $c$ are arbitrary function of $\theta$ only. Noting that $H^{12}=-i h_{34}, H^{34}=-i h_{12}, H^{13}=$ $i h_{24}$ and $H^{23}=-i h_{14}$, we find from (41)

$$
h_{34}=\left(1+a^{2}+b^{2}\right) / c .
$$


Further, one finds $F_{12}=F_{34}=-1$ and $F_{13}=F_{24}=F_{14}=F_{32}=0$. This is a good solution for the case where 123456 directions are compactified on $T^{6}$.

\subsection{New solutions}

Using (35) in (45), we have

$$
\begin{aligned}
H^{12} & =\frac{-i}{1+\frac{1}{2}|\nabla Z|^{2}} h_{34}, & H^{34} & =\frac{-i}{1+\frac{1}{2}|\nabla Z|^{2}} h_{12}, \\
H^{13}=H^{24} & =\frac{i}{1+\frac{1}{2}|\nabla Z|^{2}} h_{24}, & H^{23} & =-H^{14}=\frac{-i}{1+\frac{1}{2}|\nabla Z|^{2}} h_{14} .
\end{aligned}
$$

Combined with (41), this yields

$$
h_{34} h_{12}-h_{24}^{2}-h_{14}^{2}=1+\frac{1}{2}|\nabla Z|^{2} .
$$

One has to impose various condition on the field strengths following from the Bianchi identity. It is

$$
\epsilon^{i j k l} \partial_{j} h_{k l}=0
$$

Introducing the potential $b_{i}$ by $h_{i j}=\partial_{i} b_{j}-\partial_{j} b_{i}$, eq. (51) may be rewritten as

$$
h_{13}-h_{24}+i\left(h_{14}+h_{23}\right)=\left(\partial_{1}+i \partial_{2}\right)\left(b_{3}+i b_{4}\right)-\left(\partial_{3}+i \partial_{4}\right)\left(b_{1}+i b_{2}\right)=0 .
$$

The general solutions are given by

$$
b_{1}+i b_{2}=\left(\partial_{1}+i \partial_{2}\right)\left(G_{R}+i G_{I}\right), \quad b_{3}+i b_{4}=\left(\partial_{3}+i \partial_{4}\right)\left(G_{R}+i G_{I}\right) .
$$

Choosing the gauge $G_{R}=0$ and introducing $G=-G_{I}$, this is written as

$$
b_{i}=\epsilon^{i j} \partial_{j} G
$$

One then finds

$$
\begin{aligned}
& h_{12}=-\left(\partial_{1}^{2}+\partial_{2}^{2}\right) G, \quad h_{34}=-\left(\partial_{3}^{2}+\partial_{4}^{2}\right) G \\
& h_{13}=\left(\partial_{1} \partial_{4}-\partial_{2} \partial_{3}\right) G, \quad h_{14}=-\left(\partial_{1} \partial_{3}+\partial_{2} \partial_{4}\right) G .
\end{aligned}
$$

Similarly one has

$$
a_{i}=\epsilon^{i j} \partial_{j} K
$$


for $F_{i j}=\partial_{i} a_{j}-\partial_{j} a_{i}$. It is easy to show that $K$ giving (49) is

$$
K=\frac{1}{4}\left(\left|w_{1}\right|^{2}+\left|w_{2}\right|^{2}+|Z|^{2}\right)
$$

which is proportional to the Kähler potential.

Since (58) may be written as

$$
h_{34} h_{12}-h_{24}^{2}-h_{14}^{2}=F_{34} F_{12}-F_{13}^{2}-F_{14}^{2},
$$

there is a trivial solution $G= \pm K$. This is the solution presented in Ref. [26], which does not include any arbitrariness in the field strengths. We would like to examine whether there exist other independent solutions.

The solutions (55) and (56) of the $Z=0$ case involve three arbitrary functions of $\theta$. We expect that the similar type of solutions exist for the nonvanishing $Z$. We shall find new solutions involving the arbitrariness in the field strengths.

Let us now present our new solutions that involve arbitrary functions. Consider the case where $Z=f_{1}\left(w_{1}\right)+f_{2}\left(w_{2}\right)$ where $f_{a}$ are arbitrary holomorphic functions that depend only on $w_{a}$. For this, the metric becomes

$$
\begin{aligned}
& g_{11}=-1-f_{1}^{\prime} \bar{f}_{1}^{\prime}, \quad g_{33}=-1-f_{2}^{\prime} \bar{f}_{2}^{\prime}, \\
& g_{13}=-\frac{1}{2}\left(f_{1}^{\prime} \bar{f}_{2}^{\prime}+f_{2}^{\prime} \bar{f}_{1}^{\prime}\right), \quad g_{14}=\frac{i}{2}\left(f_{1}^{\prime} \bar{f}_{2}^{\prime}-f_{2}^{\prime} \bar{f}_{1}^{\prime}\right) .
\end{aligned}
$$

The solution involving arbitrary functions of $\theta$ then becomes

$$
\begin{aligned}
& h_{12}=-c(\theta)\left(1+f_{1}^{\prime} \bar{f}_{1}^{\prime}\right), \quad h_{34}=-\frac{1+f_{2}^{\prime} \bar{f}_{2}^{\prime}}{c(\theta)}, \\
& h_{14}=-\frac{e^{i a(\theta)}}{2} f_{1}^{\prime} \bar{f}_{2}^{\prime}-\frac{e^{-i a(\theta)}}{2} f_{2}^{\prime} \bar{f}_{1}^{\prime}, \\
& h_{13}=-i \frac{e^{i a(\theta)}}{2} f_{1}^{\prime} \bar{f}_{2}^{\prime}+i \frac{e^{-i a(\theta)}}{2} f_{2}^{\prime} \bar{f}_{1}^{\prime},
\end{aligned}
$$

One can check that the above satisfy (66) and the Bianchi identity. In fact, we find that the gauge potential (62) giving these field strengths is

$$
G=\frac{c(\theta)}{4}\left(\left|w_{1}\right|^{2}+\left|f_{1}\right|^{2}\right)+\frac{\left|w_{2}\right|^{2}+\left|f_{2}\right|^{2}}{4 c(\theta)}+\frac{e^{i a(\theta)}}{4} f_{1} \bar{f}_{2}+\frac{e^{-i a(\theta)}}{4} \bar{f}_{1} f_{2} .
$$

To complete the analysis, one may consider a small fluctuation around the solution $h_{i j}=$ $\frac{1}{2}\left(\epsilon_{i k} g_{k j}-\epsilon_{j k} g_{k i}\right)$. The small perturbation equation becomes

$$
\begin{aligned}
& \left(1+f_{1}^{\prime} \bar{f}_{1}^{\prime}\right) \partial_{w_{2}} \partial_{\bar{w}_{2}} \delta G+\left(1+f_{2}^{\prime} \bar{f}_{2}^{\prime}\right) \partial_{w_{1}} \partial_{\bar{w}_{1}} \delta G \\
& -f_{1}^{\prime} \bar{f}_{2}^{\prime} \partial_{w_{2}} \partial_{\bar{w}_{1}} \delta G-f_{2}^{\prime} \bar{f}_{1}^{\prime} \partial_{w_{1}} \partial_{\bar{w}_{2}} \delta G=0 .
\end{aligned}
$$


One can check the above deformation by $a$ and $c$ gives two independent solutions to these zeromode equations. To check if this exhaust all possibility, let us find out the number of zero modes.

\subsection{Zero-mode equations}

We first consider the simplest case where $f_{1}=f_{2}=0(Z=0)$. The zero-mode equation (70) becomes the Laplace equation in the flat four dimensions:

$$
\left(\partial_{1}^{2}+\partial_{2}^{2}+\partial_{3}^{2}+\partial_{4}^{2}\right) \delta G=0
$$

which leads to the equation

$$
\left(\partial_{1}^{2}+\partial_{2}^{2}+\partial_{3}^{2}+\partial_{4}^{2}\right) \delta h_{i j}=0
$$

together with the Bianchi identity.

For the case of $T^{4}, \delta h_{i j}$ defined on $T^{4}$ should have the absolute minimum, which contradicts the properties of Laplace equation unless $\delta h_{i j}$ 's are constant functions.

For the case of flat four plane, again $\delta h_{i j}$ should be constant if one does not allow singularity or divergences at infinity. Then one may show that there are only three independent regular solutions,

$$
\begin{aligned}
& \delta h_{12}=-\delta h_{34}=\delta c(\theta), \\
& \delta h_{13}=\delta h_{24}=\delta a(\theta), \\
& \delta h_{14}=\delta h_{32}=\delta b(\theta),
\end{aligned}
$$

where $\delta a, \delta b$, and $\delta c$ are functions of $\theta$ only. This is consistent with the full solution in (55).

Let us consider the general compact four Kähler manifold with a Kähler metric $g_{p \bar{q}}$. The equation we have to solve is the Hermitian Yang-Mills equation

$$
g^{p \bar{q}} \delta h_{p \bar{q}}=0
$$

with $\delta h_{p q}=\delta h_{\bar{p} \bar{q}}=0$. Of course the Bianchi identity

$$
d \delta h=0,
$$

has to be satisfied. Note that the Hermitian Yang-Mills equation combined with the Bianchi identity implies that

$$
d^{\dagger} \delta h=0,
$$


which follows from $g^{p \bar{q}} \nabla_{p} \delta h_{\bar{q} r}=\nabla_{r}\left(g^{p \bar{q}} \delta h_{p \bar{q}}\right)$. Therefore $\delta h$ is a harmonic $(1,1)$ form which satisfies an additional constraint of the Hermitian Yang-Mills equation (74). From the above computation, the harmonic $(1,1)$ form satisfies in general

$$
g^{p \bar{q}} \delta h_{p \bar{q}}=C
$$

where $C$ is constant. Therefore the number of zero mode, $I$, in the compact Kähler space is given by

$$
I=h_{1,1}-1
$$

where $h_{p, q}$ denotes the Hodge number of the harmonic $(p, q)$ form. For $Z=0$, the four manifold describes $T^{4}$, whose Hodge number $h_{1,1}$ is four. Thus for $Z=0, I=3$, which is consistent with the explicit construction (73).

For $S^{2} \times S^{2}$ which is Kähler, $h_{1,1}=2$ and, hence, $I=1$. More explicitly, let us use the complex coordinate $w^{1}$ and $w^{2}$ for the spheres. Then the harmonics forms are given by the volume forms $\sqrt{g_{(1)}} d w^{1} \wedge d \bar{w}^{1}$ and $\sqrt{g_{(2)}} d w^{2} \wedge d \bar{w}^{2}$. The hermitian Yang-Mills equations are satisfied only by

$$
\delta h=\sqrt{g_{(1)}} d w^{1} \wedge d \bar{w}^{1}-\sqrt{g_{(2)}} d w^{2} \wedge d \bar{w}^{2} .
$$

Therefore we find that $I=1$ by the explicit construction.

\section{Charges and Conserved Quantities}

In order to understand what microstates are possible, we should look at the configurations with various conserved quantities fixed [23]. In this section, we derive explicit expressions of these quantities and examine bounds on the shapes of the BPS configurations.

From the Chern-Simons term,

$$
\frac{1}{2} \int H \wedge C_{(3)}
$$

the M2-brane charge density extended in the $(i j)$ direction can be identified as ${ }^{1}$

$$
Q_{i j}=\frac{1}{4 \pi} \epsilon_{i j k l} \int_{0}^{2 \pi} d \theta H_{k l 5}=\frac{1}{4 \pi} \epsilon_{i j k l} \int_{0}^{2 \pi} d \theta h_{k l} \sqrt{\left|g_{55}\right|}
$$

\footnotetext{
${ }^{1}$ The actual charge here is twice of the above Chern-Simons contribution[37].
} 
where the density is the charges per unit coordinate area in the $(k l)$ directions ${ }^{2}$. To compute the angular momentum, let us first compute the linear momentum density in the $\theta$ direction. This is given by

$$
\mathcal{P}_{5}=\Pi^{0 i j} H_{5 i j}
$$

where the displacement $\Pi^{0 i j}$ is defined by

$$
\Pi^{0 i j}=\frac{\partial \mathcal{L}}{\partial \dot{A_{i j}}}=\frac{\sqrt{-g}}{2} H^{* 0 i j}=\frac{1}{4} \epsilon^{i j k l} H_{k l 5}=\frac{i \sqrt{-g}}{2} H^{i j}
$$

For the momentum density, we ignore the contributions from other fields or time dependence, which vanishes due to the BPS conditions. Using the expression for the displacement and (36), the momentum density is evaluated as

$$
\mathcal{P}_{5}=-\sqrt{g_{(4)}}\left|g_{55}\right| \frac{\sqrt{g_{(4)}}}{8} \epsilon_{i j k l} H^{i j} H^{k l}=\sqrt{g_{4}} X_{m}^{\prime} X_{m}^{\prime} .
$$

In the target space viewpoint, the momentum density along the circle direction of tube, is then given by

$$
P_{m}=\sqrt{g_{(4)}} X_{m}^{\prime}
$$

The angular-momentum density in the transverse space is given by

$$
J_{m n}=\frac{\sqrt{g_{(4)}}}{\pi} \int_{R} d X^{m} \wedge d X^{n} .
$$

where $R$ denotes the region enclosed by the arbitrary curve $\mathcal{C}$ of $X^{m}(\theta)$.

Since the angular momentum is proportional to the area, the length $L$ of the cross section of the supertube

$$
L=\frac{1}{2 \pi} \int_{0}^{2 \pi} d \theta \sqrt{X_{m}^{\prime} X_{m}^{\prime}}
$$

is bounded from below by the square root of the area [20]. When the curve is confined in the (12) space, one has the bound

$$
\left|J_{12}\right| \leq \sqrt{g_{(4)}} L^{2}
$$

Considering more general curve, one may get

$$
\left|J_{12}^{\prime}\right|+\left|J_{34}^{\prime}\right| \leq \sqrt{g_{(4)}} L^{2}
$$

\footnotetext{
${ }^{2}$ Here we consider the holomorphic four submanifold independent of $\theta$ for simplicity.
} 
where $J_{12}^{\prime}$ and $J_{34}^{\prime}$ represents the value of Cartans when only Cartans remain nonvanishing by the $S O(4)$ rotations.

The curve is also constrained by the combination of charges. The inequality is given by

$$
\sqrt{g_{(4)}} L^{2} \leq W(Q)
$$

where

$$
W(Q)=\frac{1}{8}\left|\epsilon^{i j k l} Q_{i j} Q_{k l}\right| .
$$

The proof is as follows. Let us work in the local Lorentz frame where $Q_{i j}$ is block diagonalized with only nonvanishing components $Q_{12}$ and $Q_{34}$. Then one has

$$
\begin{aligned}
\sqrt{\left|Q_{12} Q_{34}\right|} & \geq \frac{1}{2 \pi} \int_{0}^{2 \pi} d \theta \sqrt{\left|g_{55}\right|} \sqrt{\left|h_{12} h_{34}\right|} \geq \frac{1}{2 \pi} \int_{0}^{2 \pi} d \theta \sqrt{\left|g_{55}\right|} \sqrt{\left|\epsilon^{i j k l} h_{i j} h_{k l} / 8\right|} \\
& \geq\left(\sqrt{g_{(4)}}\right)^{\frac{1}{2}} \frac{1}{2 \pi} \int_{0}^{2 \pi} d \theta \sqrt{\left|g_{55}\right|} .
\end{aligned}
$$

Therefore the arbitrary curve is constrained by the conserved quantities as

$$
\left|J_{12}^{\prime}\right|+\left|J_{34}^{\prime}\right| \leq \sqrt{g_{(4)}} L^{2} \leq W(Q) .
$$

This describes the moduli fluctuation of the bosonic degrees with conserved charges and angular momentum.

For the case of $T^{6}$, one has 3 kind of simple holomorphic four cycles defined by $Z^{\alpha}=C^{\alpha}$ $(\alpha=1,2,3)$ and let us consider the case where $\mathrm{m} 5$ wraps each four cycle once. Then for each four cycle, we get the above inequality. Hence we get

$$
\left|J_{12}^{\prime}\right|+\left|J_{34}^{\prime}\right| \leq \sqrt{g_{(4)}} L^{2} \leq \operatorname{Min}\left[W\left(Q_{(1)}\right), W\left(Q_{(2)}\right), W\left(Q_{(3)}\right)\right],
$$

where $I=1,2,3$ label the three holomorphic four cycles. We get the further restriction because each cycle puts independent restrictions.

Finally the total energy of the system for the supertubes is given by

$$
E=-\int_{0}^{2 \pi} d \theta \sqrt{\left|g_{55}\right|} \int_{P} h \wedge F
$$

where $P$ denotes the holomorphic four submanifold.

\section{M-theory on a CY space}

In this section, we would like to consider the embedding of m5 and M2 system into a CY 3-fold instead of $T^{6}$. As before, the m5-branes wrap four cycles of the $\mathrm{CY}$ and the remaining one direction forms an arbitrary curve in 789 Ł directions which is again taken to be flat. 


\subsection{Killing spinor}

Before describing the m5-brane problem, let us first describe the Killing spinor of M-theory CY compactification. In this compactification, the remaining Killing spinor has eight real components corresponding to $N=2$ supersymmetries. To describe the nature of this spinor, we start from 32 component complex spinor which has twice as many components of the maximal Killing spinor in eleven dimensions. Further we introduce a vielbein for the CY space whose only purely holomorphic or antiholomorphic components are nonvanishing. This gauge choice of vielbein is possible whenever the space is hermitian. The CY metric is then given by $g_{\alpha \bar{\beta}}=E_{\alpha}^{\gamma} E_{\bar{\beta}}^{\bar{\gamma}}$ where the vielbein $E_{\alpha}^{\beta}$ satisfies $E_{\bar{\alpha}}^{\bar{\beta}}=\left(E_{\alpha}^{\beta}\right)^{*}$ with $\alpha, \beta, \gamma=1,2,3$. The eleven-dimensional gamma matrices are taken to be pure imaginary.

The remaining component of the Killing spinor is constructed as follows. Consider a spinor satisfying the projection

$$
\tilde{\Gamma}_{\bar{\alpha}} \epsilon_{+}=0, \quad \text { for } \alpha=1,2,3,
$$

where we define $\epsilon_{ \pm}$by the projection

$$
\Gamma_{(7)} \epsilon_{ \pm}= \pm \epsilon_{ \pm}
$$

with $\Gamma_{(7)}=i \Gamma_{1} \Gamma_{2} \cdots \Gamma_{6}$

The gamma matrices $\tilde{\Gamma}_{\bar{\alpha}}$ is defined by $\frac{1}{2}\left(\Gamma_{2 \alpha-1}+i \Gamma_{2 \alpha}\right)$ whereas $\tilde{\Gamma}_{\alpha}$ by $\frac{1}{2}\left(\Gamma_{2 \alpha-1}-i \Gamma_{2 \alpha}\right)$. At this stage, the total 32 complex space is projected by the factor of $1 / 4$. We then impose the two conditions

$$
\begin{aligned}
& \tilde{\Gamma}_{\alpha} \tilde{\Gamma}_{\beta} \tilde{\Gamma}_{\gamma} \epsilon_{+}=\epsilon_{\alpha \beta \gamma} \epsilon_{-}, \\
& \epsilon_{-}=\epsilon_{+}^{*},
\end{aligned}
$$

where the first choice relies on the existence of the nowhere vanishing holomorphic $(3,0)$ form. By these two conditions, the space of spinor is further projected down by the factor of $1 / 4$. The real eight-dimensional spinor is constructed by

$$
\epsilon_{C Y}=c \epsilon_{+}+c^{*} \epsilon_{-}
$$

where $c$ is an arbitrary constant. This is the Killing spinor we use for the study of BPS configuration of the CY compactification.

The induced vielbein on the worldvolume

$$
e_{i}^{a}=\frac{\partial Y^{b}}{\partial \sigma^{i}} E_{b}^{a},
$$


relates the gamma matrices as

$$
\gamma_{i}=e_{i}^{a} \Gamma_{a}=\partial_{i} Y^{a} E_{a}^{b} \Gamma_{b}, \quad \gamma_{0}=\Gamma_{0}, \quad \gamma_{5}=X^{m \prime} \Gamma_{m}
$$

and the induced metric

$$
g_{00}=1, \quad g_{i j}=\partial_{i} Y^{a} \partial_{j} Y^{b} g_{a b}, \quad g_{55}=X^{m \prime} X_{m}^{\prime}
$$

Then the kappa symmetry condition is given by the same expressions as those in (5) and (6).

The analysis of the BPS equations of section 2 goes through if we choose a projection

$$
\Gamma_{0} \epsilon_{+}=-\epsilon_{+},
$$

which leads to $P_{1} \epsilon_{C Y}=P_{2} \epsilon_{C Y}=P_{3} \epsilon_{C Y}=\epsilon_{C Y}$. Further half of the supersymmetries are projected out and only four real supersymmetries remain unbroken. The BPS equations then become those in $(18)-(25)$.

\subsection{Analysis of the BPS equations}

To solve our BPS equations, we make again further gauge choice

$$
\left(Y^{1}, Y^{2}, Y^{3}, Y^{4}\right)=\left(\sigma^{1}, \sigma^{2}, \sigma^{3}, \sigma^{4}\right)
$$

and introduce the complex coordinate

$$
Z=Y^{5}+i Y^{6}=Z^{3} ; \quad w_{1}=\sigma^{1}+i \sigma^{2}=Z^{1}, \quad w_{2}=\sigma^{3}+i \sigma^{4}=Z^{2},
$$

Then eq. (20) may be written as

$$
\epsilon_{a b} f^{a b \bar{\alpha} \bar{\beta}}=0
$$

This equation is solved by any holomorphic function $Z$ satisfying

$$
\partial_{\bar{w}_{1}} Z=\partial_{\bar{w}_{2}} Z=0 \text {. }
$$

To show this, let us solve eq. (106) for $\bar{\alpha}=2$ and $\bar{\beta}=3$. The equation becomes

$$
\epsilon_{i j k l} \partial_{i} Z^{\alpha} \partial_{j} \bar{Z}^{\bar{\beta}} \partial_{k} \bar{Z}^{\bar{\gamma}} \partial_{l} \bar{Z}^{\bar{\delta}} E_{\alpha}^{1} E_{\bar{\beta}}^{\overline{1}} E_{\bar{\gamma}}^{\overline{2}} E_{\bar{\delta}}^{\overline{3}}=\epsilon_{i j k l} \partial_{i} Z^{\alpha} \partial_{j} \bar{Z}^{\overline{1}} \partial_{k} \bar{Z}^{\overline{2}} \partial_{l} \bar{Z}^{\overline{3}} \operatorname{det}\left(E_{\bar{\delta}}^{\bar{\gamma}}\right) E_{\alpha}^{1}=0
$$

Considering also $\bar{\alpha}, \bar{\beta}=1,2$ and $\bar{\alpha}, \bar{\beta}=1,3$ and noting $\operatorname{det}\left(E_{\bar{\delta}}^{\bar{\gamma}}\right) \neq 0$, one gets

$$
\epsilon_{i j k l} \partial_{i} Z^{\alpha} \partial_{j} \bar{Z}^{\overline{1}} \partial_{k} \bar{Z}^{\overline{2}} \partial_{l} \bar{Z}^{\overline{3}}=0,
$$


which leads to the holomorphicity condition (107). Eq. (24) is again solved for

$$
H^{w_{1} w_{2}}=h_{w_{1} w_{2}}=0
$$

and eqs. (21) and (22) imply

$$
H^{i 5}=H^{05}=0 .
$$

Eq. (19) can be written as

$$
\frac{1}{8} \sqrt{g_{(4)}} \epsilon_{i j k l} H^{i j} H^{k l}=-1
$$

and the last BPS equation (25) follows automatically as before. The constraint equation (42) is again solved by (50). Thus we are left with the final equation

$$
\frac{1}{8 \sqrt{g_{(4)}}} \epsilon^{i j k l} h_{i j} h_{k l}=1
$$

with the Bianchi identity $d h=0$. One of the trivial solution of the equation is given by

$$
h=i k,
$$

where $k$ is the Kähler two form.

The most general solutions are described as follows. m5 wraps holomorphic four cycles, which is classified by the the Hodge number $h^{1,1}(M)$ of the CY manifold $M$. Or the four cycles are described by codimension 1 hypersurface defined as a zero locus of holomorphic function on $M$. This precisely corresponds to divisor of the CY manifold $M$. Each section of line bundle $\mathcal{L}$ on $M$ is in one-to-one correspondence with divisor $P$. The deformation space of the divisor is given by $2\left(h^{0}(M, \mathcal{L}(P))-1\right)=2 h^{2,0}(P)[38]$.

For any such four cycles $P$ in $M$, one has to solve the nonlinear instanton equation (113). The full nonlinear analysis of the equations seems very complicated. But one may find the dimension of the solution space by linearizing the equation around the trivial solution $h=i k+\delta h$. $\delta h$ satisfies the Hermitian Yang-Mills equation. The solutions are harmonic $(1,1)$ form on $P$ with one extra constraint that the harmonic $(1,1)$ form should be anti-selfdual. The only selfdual harmonic $(1,1)$ form is the Kähler two form. The number of dimensions of anti-selfdual harmonic $(1,1)$ form is thus $h_{-}^{1,1}(P)=h^{1,1}(P)-1$.

Thus for each cohomology class of $H^{1,1}(M)$, most general solutions involves $2 h^{2,0}(P)+$ $h^{1,1}(P)-1=b_{2}(P)-1$ arbitrary functions of $\theta$ in addition to the arbitrary curve in $789 \curvearrowleft$ directions, where $b_{2}$ is the Betti number. The curve involves only 3 physical degrees of the 
transverse fluctuation because of the reparametrization invariance along $\theta$ directions. Therefore the total number of degrees involved with the arbitrary function of $\theta$ is given by $b_{2}(P)+2$, which is related to the Euler number by

$$
b_{2}(P)+2=\chi(P)+4 b_{1}(P) .
$$

If the divisor is very ample, one has $b_{1}(P)=b_{1}(M)$ and then $b_{2}(P)+2=\chi(P)$. (Here we use the property $b_{1}(M)=0$ for a $\mathrm{CY}$ manifold of exactly $\mathrm{SU}(3)$ holonomy and not its subgroup.) The Euler number is evaluated as

$$
\chi(P)=\int_{M}\left(P^{3}+P c_{2}(M)\right),
$$

where $P$ is the $(1,1)$ form of $M$ dual to the divisor and $c_{2}$ is the second Chern class.

For the divisor $P=T^{4}$ of $M=T^{6}$, the above $\chi(P)=0$ but $b_{1}(P)=b_{3}(P)=2$. Hence the number of moduli is 8 for the $T^{4}$; three for the transverse fluctuation in the $789 \natural$ space, two for the $T^{4}$ moduli in $T^{6}$ and three for the flux moduli as we verified explicitly.

\section{Supertubes with 4 M2-Charges}

In this section, we consider the M-theory compactified on $T^{8}$ (or CY 4-fold) as a generalization of the 3 M2-charge case. In this compactification, we consider the configuration involving four M2-branes extended along (12), (34), (56), and (78) spatial directions of the tangent space. This is what we call 4-charge supertubes in M-theory.

The setting to obtain the BPS equations from (2) to (10) are unchanged with a few modifications. The indices $a, b, c$ run now from 1 to 8 and $m, n$ stand for 9 and $\downarrow$. The relevant projection operator becomes now

$$
\epsilon=P_{1} P_{2} P_{3} P_{4} \epsilon_{0}
$$

where $P_{1,2,3}$ are the same as before and $P_{4}=\frac{1+i \Gamma_{078}}{2}$.

With the gauge choice $a=t, H^{i 0}=H^{50}=0$ automatically follow from the definition (44). The $\kappa$-symmetry condition gives

$$
\begin{aligned}
\Gamma \epsilon & =\frac{\sqrt{-g}}{\sqrt{-(g+i H)}}\left(\frac{1}{\sqrt{-g}} \Gamma_{0} \gamma_{5} \Gamma_{a b c d} f^{a b c d}+\frac{1}{2} H^{i j} \gamma_{i j} \Gamma_{0}+H^{i 5} \gamma_{i} \gamma_{5} \Gamma_{0}\right. \\
& \left.-\frac{1}{8} \sqrt{-g} \epsilon_{i j k l} H^{i j} H^{k l} \gamma^{5} \Gamma_{0}-\frac{1}{2} \sqrt{-g} \epsilon_{i j k l} H^{5 i} H^{j k} \gamma^{l} \Gamma_{0}\right) \epsilon=-\epsilon .
\end{aligned}
$$

The 3rd term in the big parenthesis cannot be canceled by others and, consequently, $H^{i 5}=0$. 
With the help of the projection operators, the first term in the big parenthesis may be arranged as

$$
\begin{aligned}
\Gamma_{a b c d} f^{a b c d} \epsilon & =3 f^{\bar{\alpha} \bar{\beta} \gamma \delta} \delta_{\bar{\alpha} \delta} \delta_{\bar{\beta} \gamma} \epsilon+\left(f^{\alpha \beta \gamma \delta} \tilde{\Gamma}_{\alpha} \tilde{\Gamma}_{\beta} \tilde{\Gamma}_{\gamma} \tilde{\Gamma}_{\delta}-6 f^{\bar{\alpha} \beta \gamma \delta} \delta_{\bar{\alpha} \beta} \tilde{\Gamma}_{\gamma} \tilde{\Gamma}_{\delta}\right) \eta_{+} \\
& +\left(f^{\bar{\alpha} \bar{\beta} \bar{\gamma} \bar{\delta}} \tilde{\Gamma}_{\bar{\alpha}} \tilde{\Gamma}_{\bar{\beta}} \tilde{\Gamma}_{\bar{\gamma}} \tilde{\Gamma}_{\bar{\delta}}-6 f^{\alpha \bar{\beta} \bar{\gamma} \bar{\delta}} \delta_{\bar{\beta} \alpha} \tilde{\Gamma}_{\bar{\gamma}} \tilde{\Gamma}_{\bar{\delta}}\right) \eta_{-}
\end{aligned}
$$

where $\eta_{ \pm}=\frac{1 \pm \Gamma_{0}}{2} \epsilon$, and $\alpha, \beta, \gamma, \delta$ are holomorphic indices running from 1 to 4 . Thus one is lead to the BPS equations,

$$
f^{\alpha \beta \gamma \delta} \epsilon_{\alpha \beta \gamma \delta}=0, f^{\bar{\alpha} \beta \gamma \delta} \delta_{\bar{\alpha} \beta}=0 .
$$

The holomorphic target space coordinates are given by $Z^{\alpha}=Y^{2 \alpha-1}+i Y^{2 \alpha}$. Again we choose the gauge $w_{1}=\sigma^{1}+i \sigma^{2}=Z^{1}$ and $w_{2}=\sigma^{3}+i \sigma^{4}=Z^{2}$. Then the above equation implies simply that $Z^{3}$ and $Z^{4}$ are holomorphic. Namely m5 is wrapping the holomorphic four cycles.

The coefficient of $\gamma_{5} \Gamma_{0}$ has to be zero. After some algebra, this leads to the BPS equation

$$
\frac{1}{8} \sqrt{g_{(4)}} \epsilon_{i j k l} H^{i j} H^{k l}=-1,
$$

which agrees with the expression of the 3-charge case.

From the second term in the big parenthesis, one gets the BPS equation

$$
H^{w_{2} w_{2}}=H^{\bar{w}_{2} \bar{w}_{2}}=0 .
$$

Then the final remaining equation requires

$$
g_{p \bar{q}} H^{p \bar{q}}=\frac{\sqrt{-(g+i H)}}{\sqrt{-g}},
$$

where $p, q(\bar{p}, \bar{q})$ are the worldvolume (anti) holomorphic indices running over 1,2 . This last condition again automatically follows from the above BPS equations.

Hence the $\mathrm{m} 5$ are again described by the holomorphic four cycles of the eight manifold $M$. Then the worldvolume flux of $(1,1)$ type satisfying $(121)$ may be turned on.

\section{Conclusion}

In this paper we have first discussed the 3-charge supertubes in the M-theory compactified on M (that may be $T^{6}$ or CY 3-fold). The system under consideration consists of M2 branes extended along the (12), (34) and (56) tangent space directions of the internal manifold $M$. The four spatial directions of m5-branes wraps four cycles of $M$ and the remaining spatial direction forms an arbitrary curve in the space transverse to the internal manifold. 
By analyzing the m5-brane action in detail, we have obtained the BPS equations governing the dynamics of the supertubes. The nature of their solutions are fully understood. For some particular cases, we have worked out the solutions explicitly.

The most general supertube solutions involving 3 charges may be described as follows. The four directions of m5 may wrap any holomorphic four cycle $P$, which is described by the zero locus of the holomorphic function over $M$. The holomorphic four cycles are dual to $(1,1)$ type harmonic two forms restricted to the Kähler cone. As said in the above, the remaining spatial direction forms an arbitrary curve in the transverse space. The worldvolume flux may be turned on, which has to be $(1,1)$ type in $P$. This two form flux has to satisfy the nonlinear instanton equation.

The $P$ may be deformed smoothly inside $M$ and the dimension of this deformation space is given by $2 h^{2,0}(P)$. And also the solution space dimensions of the instanton equation of the two form are shown to be given by $h^{1,1}(P)-1$. Then the shape of $P$ and the two form flux may vary over the moduli space as we move along the curve directions as a function of $\theta$.

With fixed energy, angular momentum and other conserved charges, the number of wraps of the cycles by m5 may be arbitrary as the m5-brane charges are not conserved due to its dipole nature. However, by the continuity of the deformation along the curve direction, the number of wraps cannot be changed along the curve directions because the number is topological. In this sense, we expect that, in the moduli space of supertubes, the number of wraps of $P$ labels superselection sectors of the whole moduli space.

We have also included brief discussion of the 4-charge generalization.

After analyzing the most general configuration, the natural question is on the degeneracy of the configurations with fixed conserved charges. This investigation will lead to the microscopic understanding of the entropy of supertubes. Since the system has an infinite number of degrees classically, one has to quantize the moduli fluctuations properly. The contribution of the fermions should also be included if there are any. In fact this problem has been considered in Ref. [40] but we would like to be more explicit and fill some possible gaps. This will be our main subject of the further investigations. 


\section{Acknowledgment}

We would like to thank A. Tsuchiya for valuable discussions at the early stage of this work. D.B. is supported in part by KOSEF ABRL R14-2003-012-01002-0, KOSEF R01-2003-000-10319-0 and KOSEF SRC CQUeST R11-2005-021. The work of NO was supported in part by the Grant-in-Aid for Scientific Research Fund of the JSPS No. 16540250.

\section{References}

[1] D. Mateos and P. K. Townsend, "Supertubes," Phys. Rev. Lett. 87 (2001) 011602 [arXiv:hep-th/0103030].

[2] D. Bak and K. Lee, "Noncommutative supersymmetric tubes," Phys. Lett. B 509 (2001) 168 [arXiv:hep-th/0103148].

[3] R. Emparan, D. Mateos and P. K. Townsend, "Supergravity supertubes," JHEP 0107 (2001) 011 [arXiv:hep-th/0106012].

[4] D. Bak and S. W. Kim, "Junctions of supersymmetric tubes," Nucl. Phys. B 622 (2002) 95 [arXiv:hep-th/0108207].

[5] D. Bak and A. Karch, "Supersymmetric brane-antibrane configurations," Nucl. Phys. B 626 (2002) 165 [arXiv:hep-th/0110039].

[6] D. Bak and N. Ohta, "Supersymmetric D2 anti-D2 strings," Phys. Lett. B 527 (2002) 131 [arXiv:hep-th/0112034].

[7] D. Mateos, S. Ng and P. K. Townsend, "Tachyons, supertubes and brane/anti-brane systems," JHEP 0203 (2002) 016 [arXiv:hep-th/0112054].

[8] D. Mateos, S. Ng and P. K. Townsend, "Supercurves," Phys. Lett. B 538 (2002) 366 [arXiv:hep-th/0204062].

[9] M. Kruczenski, R. C. Myers, A. W. Peet and D. J. Winters, "Aspects of supertubes," JHEP 0205 (2002) 017 [arXiv:hep-th/0204103].

[10] Y. Hyakutake and N. Ohta, "Supertubes and supercurves from M-ribbons," Phys. Lett. B 539 (2002) 153 [arXiv:hep-th/0204161]. 
[11] J. H. Cho and P. Oh, "Elliptic supertube and a Bogomol'nyi-Prasad-Sommerfield D2-braneanti-D2-brane pair," Phys. Rev. D 65 (2002) 121901.

[12] D. Bak, N. Ohta and M. M. Sheikh-Jabbari, "Supersymmetric brane anti-brane systems: Matrix model description, stability and decoupling limits," JHEP 0209 (2002) 048 [arXiv:hep-th/0205265].

[13] D. Bak and K. M. Lee, "Supertubes connecting D4 branes," Phys. Lett. B 544 (2002) 329 [arXiv:hep-th/0206185].

[14] N. Drukker, B. Fiol and J. Simon, "Goedel's universe in a supertube shroud," Phys. Rev. Lett. 91 (2003) 231601 [arXiv:hep-th/0306057].

[15] S. Kim and K. Lee, "Dyonic instanton as supertube between D4 branes," JHEP 0309 (2003) 035 [arXiv:hep-th/0307048].

[16] C. j. Kim, Y. b. Kim, O. K. Kwon and P. Yi, "Tachyon tube and supertube," JHEP 0309 (2003) 042 [arXiv:hep-th/0307184].

[17] H. Takayanagi, "Boundary states for supertubes in flat spacetime and Goedel universe," JHEP 0312 (2003) 011 [arXiv:hep-th/0309135].

[18] H. Elvang and R. Emparan, "Black rings, supertubes, and a stringy resolution of black hole non-uniqueness," JHEP 0311 (2003) 035 [arXiv:hep-th/0310008].

[19] B. C. Palmer and D. Marolf, "Counting supertubes," JHEP 0406 (2004) 028 [arXiv:hepth/0403025].

[20] D. Bak, Y. Hyakutake and N. Ohta, "Phase moduli space of supertubes," Nucl. Phys. B 696 (2004) 251 [arXiv:hep-th/0404104].

[21] N. Drukker, "Supertube domain-walls and elimination of closed time-like curves in string theory," Phys. Rev. D 70 (2004) 084031 [arXiv:hep-th/0404239].

[22] W. H. Huang, "Tachyon tube on non BPS D-branes," JHEP 0408 (2004) 060 [arXiv:hepth/0407081].

[23] D. Bak, Y. Hyakutake, S. Kim and N. Ohta, "A geometric look on the microstates of supertubes," Nucl. Phys. B 712 (2005) 115 [arXiv:hep-th/0407253]. 
[24] I. Bena and P. Kraus, "Three charge supertubes and black hole hair," arXiv:hepth/0402144.

[25] I. Bena and N. P. Warner, "One ring to rule them all ... and in the darkness bind them?," arXiv:hep-th/0408106.

[26] H. Elvang, R. Emparan, D. Mateos and H. S. Reall, "Supersymmetric black rings and three-charge supertubes," Phys. Rev. D 71 (2005) 024033 [arXiv:hep-th/0408120].

[27] J. P. Gauntlett and J. B. Gutowski, "General concentric black rings," Phys. Rev. D 71 (2005) 045002 [arXiv:hep-th/0408122].

[28] I. Bena, C. W. Wang and N. P. Warner, "Black rings with varying charge density," arXiv:hep-th/0411072.

[29] P. G. Shepard, "Bloch waves and fuzzy cylinders: 1/4-BPS solutions of matrix theory," arXiv:hep-th/0510127.

[30] O. Lunin and S. D. Mathur, "AdS/CFT duality and the black hole information paradox," Nucl. Phys. B 623 (2002) 342 [arXiv:hep-th/0109154].

[31] O. Lunin, J. Maldacena and L. Maoz, "Gravity solutions for the D1-D5 system with angular momentum," arXiv:hep-th/0212210.

[32] S. D. Mathur, A. Saxena and Y. K. Srivastava, "Constructing 'hair' for the three charge hole," Nucl. Phys. B 680 (2004) 415 [arXiv:hep-th/0311092].

[33] O. Lunin, "Adding momentum to D1-D5 system," JHEP 0404 (2004) 054 [arXiv:hepth/0404006].

[34] S. Giusto, S. D. Mathur and A. Saxena, "Dual geometries for a set of 3-charge microstates," Nucl. Phys. B 701 (2004) 357 [arXiv:hep-th/0405017]; "3-charge geometries and their CFT duals," Nucl. Phys. B 710 (2005) 425 [arXiv:hep-th/0406103].

[35] I. A. Bandos, K. Lechner, A. Nurmagambetov, P. Pasti, D. P. Sorokin and M. Tonin, "Covariant action for the super-five-brane of M-theory," Phys. Rev. Lett. 78 (1997) 4332 [arXiv:hep-th/9701149].

[36] P. Pasti, D. P. Sorokin and M. Tonin, "Covariant action for a D = 11 five-brane with the chiral field," Phys. Lett. B 398 (1997) 41 [arXiv:hep-th/9701037]. 
[37] D. P. Sorokin and P. K. Townsend, Phys. Lett. B 412 (1997) 265 [arXiv:hep-th/9708003].

[38] J. M. Maldacena, A. Strominger and E. Witten, "Black hole entropy in M-theory," JHEP 9712, 002 (1997) [arXiv:hep-th/9711053].

[39] M. Marino, R. Minasian, G. W. Moore and A. Strominger, "Nonlinear instantons from supersymmetric p-branes," JHEP 0001 (2000) 005 [arXiv:hep-th/9911206].

[40] M. Cyrier, M. Guica, D. Mateos and A. Strominger, "Microscopic entropy of the black ring," Phys. Rev. Lett. 94 (2005) 191601 [arXiv:hep-th/0411187]. 